\title{
Cost differences in VHA acute and subacute rehabilitation units: Implications for VHA resource planning
}

\author{
W. Bruce Vogel, PhD; ${ }^{1-2}$ Tracey E. Barnett, PhD;1,3* Dean Reker, PhD $^{4}$ \\ ${ }^{1}$ Research Health Scientist, Veterans Health Administration (VHA) Rehabilitation Outcomes Research Enhancement \\ Award Program, Gainesville, FL; ${ }^{2}$ Department of Epidemiology and Health Policy Research, College of Medicine, \\ University of Florida, Gainesville, FL; ${ }^{3}$ Department of Behavioral Science and Community Health, College of Public \\ Health and Health Professions, University of Florida, Gainesville, FL; ${ }^{4}$ VHA Information Resource Center, Chicago, \\ $I L$; and Department of Veterans Affairs Medical Center, Kansas City, KS
}

\begin{abstract}
Within the Veterans Health Administration (VHA), the top tier of postacute rehabilitation care is provided in acute rehabilitation bedservice units (ARBUs). The next level of care is provided in subacute rehabilitation bedservice units (SRBUs). We fitted reduced-form and structural models to explain VHA cost differences between ARBUs and SRBUs across time and for the individual cost components. We included sociodemographic variables, time since stroke onset, care facility, and the Functional Independence Measure at admission as explanatory variables. The multivariable results indicate that total index stay costs are lower in ARBUs by almost $\$ 6,000$ (or approximately 25\%) compared with SRBUs. Moreover, the lower costs observed in ARBUs in this study combined with the higher rates of guideline compliance and improved outcomes in ARBUs found in previous work suggest that stroke rehabilitation in an ARBU may be more cost-effective than stroke rehabilitation in an SRBU.
\end{abstract}

Key words: acute units/subacute units, cost, functional status, guidelines, lengths of stay, outcomes, planning, rehabilitation, stroke, VHA.

\section{INTRODUCTION}

Stroke is the second most frequent cause of death and the leading cause of long-term disability in the United States [1-2]. More than 700,000 Americans experience a new or recurrent stroke each year, with estimated direct and indirect costs exceeding $\$ 59$ billion [3]. Within the Veterans Health Administration (VHA), 5,000 to 6,000 inpatient stroke discharges occur annually, with annual direct inpatient and outpatient costs of more than $\$ 300$ million [4]. While the costs of acute stroke care have been studied previously [5], the costs of stroke rehabilitation have received considerably less attention [6-7]. In particular, little is known about how stroke rehabilitation costs vary across different rehabilitation settings, especially within the VHA. This lack of knowledge is surprising given the dramatic shifts over the past 10 to 15 years in the structure of VHA stroke rehabilitation, as explained in the following section. This article begins to fill this gap in our knowledge by comparing the costs of stroke rehabilitation in acute rehabilitation units to the costs of stroke rehabilitation in subacute rehabilitation

Abbreviations: ARBU = acute rehabilitation bedservice unit, DSS NDE $=$ Decision Support System National Data Extract, FIM $=$ Functional Independence Measure, FY = fiscal year, OLS = ordinary least squares, SRBU = subacute rehabilitation bedservice unit, VA = Department of Veterans Affairs, VHA = Veterans Health Administration.

*Address all correspondence to Tracey E. Barnett, PhD; Department of Veterans Affairs-Research, 1601 SW Archer Road RORC (151B), Gainesville, FL 32608; 352376-1611; fax: 352-271-4540. Email: tracey.barnett@va.gov DOI:10.1682/JRRD.2009.07.0094 
units. In so doing, we uncover surprising results that have significant implications for recent structural changes in VHA rehabilitation.

\section{BACKGROUND}

Multiple treatment settings exist for postacute stroke rehabilitation care within the VHA. The top tier of postacute rehabilitation care is provided in acute rehabilitation bedservice units (ARBUs). There are currently 31 such units in the VHA nationwide. The next level of care is provided in subacute rehabilitation bedservice units (SRBUs), with currently 24 such units nationwide. ARBUs and SRBUs share similar characteristics: they both have dedicated beds and staff and provide interdisciplinary care under a team approach. ARBUs tend to be housed in acute medical facilities (hospitals), while SRBUs tend to be housed in long-term care settings (intermediate care bedservices or nursing homes).

Beginning in 1995, the VHA undertook a major restructuring of its healthcare services under its new "Prescription for Change" initiative [8]. This initiative was designed to modernize the VHA healthcare system by (1) adopting managed care principles that emphasized the health of a well-defined enrolled population and (2) increasing the VHA's reliance on nonhospital services in place of the VHA's traditional emphasis on inpatient care. Over the next decade, the structure of VHA rehabilitation services changed dramatically: from 1995 to 2003, the number of ARBUs decreased from 59 to 31 (a $47 \%$ reduction), while the number of SRBUs increased from 0 to 24 .

Despite this substantial structural change, only limited research has examined whether differences in the processes of care and outcomes exist between ARBUs and SRBUs [9].* This limited research suggests that guideline compliance (see more information below) and possibly outcomes (as measured by functional status) are slightly higher in ARBUs than SRBUs. However, no study to date has compared the costs of care in ARBUs and SRBUs. Such information on costs is essential to

\footnotetext{
* Reker DM, Duncan PW. The process and outcomes of stroke care in the VHA. Department of Veterans Affairs, Veterans Health Administration, Rehabilitation Research and Development Service, grant O3131R; 2006.
}

begin to draw conclusions about the comparative costeffectiveness of rehabilitation in ARBUs versus SRBUs.

Guideline compliance is important to assess, as was done by Duncan et al. [10] and described in detail by LaClair et al. [11]. Briefly, processes of care dimensions were created to evaluate both acute and postacute stroke care received by patients at 11 Department of Veterans Affairs (VA) hospital sites. Eight distinct dimensions of care processes emerged for acute stroke care, while postacute stroke care yielded eleven dimensions of care processes [10-11]. Chart reviews were conducted, and the committee members who developed the original guidelines validated the dimension criteria. The committee created a weighting algorithm [10] that allowed calculation of compliance scores ranging from 0 to 100 , which we use in this work.

\section{STUDY OBJECTIVES}

We sought to answer several questions concerning stroke costs in ARBUs compared with SRBUs:

1. What are the short-term and long-term VHA total costs of care associated with rehabilitation in ARBUs compared with SRBUs? Do VHA treatment costs (index rehabilitation stay; short-term, long-term, and total costs) differ across rehabilitation unit types (ARBUs vs SRBUs) after controlling for those patient characteristics that likely influence treatment costs?

2. How do stroke guideline compliance rates and patient length of stay mediate the observed relationship between rehabilitation unit type and VHA treatment costs in item 1 above? What are the structural effects (both direct and indirect) that influence cost differences associated with rehabilitation in ARBUs compared with SRBUs?

3. Which components of VHA treatment costs (nursing, surgery, laboratory, radiology, pharmacy, and all other costs) are most responsible for the observed differences in treatment costs between ARBUs and SRBUs?

\section{METHODS}

\section{Study Design and Data Sources}

We examined costs, length of stay, and guideline compliance as endogenous (or dependent) variables in this study. We also included numerous exogenous (or independent) 
variables that might influence costs, length of stay, and guideline compliance, including age (in years), marital status (married/not married), race (white/nonwhite), impairment code (left-body involvement, right-body involvement, bilateral movement, no paresis, or other stroke), days from stroke onset to rehabilitation admission (including any days comprising an acute stroke inpatient stay), facility (the admitting VA medical center), and admission motor and cognitive Functional Independence Measure (FIM) scores [12] to control for the patient's functional status at admission.

Data for this study included patient clinical and demographic data from an evaluation of telephone versus in-person administration of the Stroke Impact Scale, a widely tested and validated instrument designed to measure the health status of patients after a stroke [10]. ${ }^{*}$ This evaluation assembled a retrospective cohort of 483 postacute stroke patients treated in 27 ARBUs and SRBUs at 23 participating VA medical centers in fiscal years (FYs) 2002 and 2003. Patients were identified with use of national VHA administrative data taken from the Functional Status Outcomes Database, the VHA's adaptation of the Uniform Data System for Medical Rehabilitation [13]. Stroke diagnosis was confirmed by review of electronic medical records. No restrictions were placed on patient inclusion in the study samples regarding sex, race, age, or socioeconomic status.

We merged these clinical and demographic data with patient-specific inpatient and outpatient cost data from the VHA Decision Support System National Data Extract (DSS NDE) for FY2002 to 2005 [14-15]. We divided patient-specific costs into four time periods covering (1) the index rehabilitation stay, (2) short-term costs (index rehabilitation stay and 0-3 months poststroke), (3) longterm costs (3-24 months poststroke), and (4) total 2-year poststroke costs of care.

Our analyses examined total costs as opposed to rehabilitation-specific costs for two reasons: (1) isolating costs that can be attributed specifically to rehabilitation in the Decision Support System is difficult and imprecise and (2) capturing any "spillover" effects that rehabilitation services may have had on non-rehabilitation services

\footnotetext{
*Reker DM, Duncan PW. The process and outcomes of stroke care in the VHA. Department of Veterans Affairs, Veterans Health Administration, Rehabilitation Research and Development Service, grant O3131R; 2006.
}

could be important in measuring the full impact of rehabilitation on total VHA resource use.

\section{Data Analysis}

We measured the total effect of type of rehabilitation unit (ARBU vs SRBU) on VHA costs as well as the direct and indirect pathways by which this total effect is achieved. To this end, we included the length of the VHA index rehabilitation stay and the poststroke rehabilitation guideline compliance score [11] as dependent variables along with costs (VHA index rehabilitation stay refers to the time period from admission to discharge in either an ARBU or SRBU following the subject stroke). We allowed the type of rehabilitation unit (acute vs subacute) to have direct effects on all three dependent variables and allowed length of stay and guideline compliance in turn to have direct effects on costs. Recognizing that longer rehabilitation stays may allow time for higher rates of compliance with poststroke guidelines, we also included length of stay as an explanatory variable for guideline compliance.

This approach yields three indirect pathways whereby type of rehabilitation unit can affect index stay costs: (1) type of unit can influence length of stay, which in turn can influence costs; (2) type of unit can influence guideline compliance, which in turn can influence costs; and (3) type of unit can influence length of stay, which influences guideline compliance, which influences costs.

We estimated reduced-form regression models of VHA costs for the (1) index rehabilitation stay, (2) shortterm (0-3 months) costs, (3) long-term (3-24 months) costs, and (4) total 2-year costs. By reduced form, we refer to regression models in which only exogenous variables are included among the regressors to ensure that the total effect (as opposed to only the direct effect) of each regressor is measured. This required excluding endogenous variables (such as length of stay and guideline compliance) that are determined simultaneously with costs. Because the type of rehabilitation unit at a facility (ARBU vs SRBU) is fixed in the short run, type of rehabilitation unit was considered exogenous for purposes of this study.

We followed the cost estimation strategy outlined by Manning and Mullahy [16] for choosing between ordinary least squares (OLS) and generalized least squares with Poisson, gamma, or inverse Gaussian distributions. This involved testing for residual kurtosis and running Park's test for determining the functional form of any heteroskedasticity [17]. 
We then estimated the structural models to further understand the pathways through which type of rehabilitation unit affects VHA costs. This was done by estimation of separate equations for length of stay and guideline compliance and inclusion of both these variables in the cost model. In so doing, we were able to separate the total effects of type of rehabilitation unit into direct and indirect effects so as to measure the relative magnitudes of the pathways by which type of rehabilitation unit influences VHA costs.

We also estimated reduced-form regressions separately for the nursing, laboratory, pharmacy, surgical, and all other cost components associated with the index rehabilitation stay to identify the source(s) of any difference in overall VHA costs.

\section{RESULTS}

Table 1 presents the descriptive statistics for our sample of patients treated in ARBUs and patients treated in SRBUs. Overall, patients seen in ARBUs and patients seen in SRBUs do not differ markedly on the measures used in this research. Mean length of stay for ARBU patients (21.7 days) were shorter than mean length of stay for SRBU patients (28.6 days). In addition, mean admission cognitive and total FIM scores were somewhat higher (a higher FIM score translates to better function) for patients seen in ARBUs than for patients seen in SRBUs (24.21 vs 21.30 cognitive, 71.08 vs 65.38 total, respectively).

Table 2 presents the results for our reduced-form regression models for total index stay costs after controlling for type of bed unit (ARBU vs SRBU), impairment code, onset to admission, admission motor and cognitive FIM scores, and demographics. These models measure the total effects (direct plus indirect pathways) of the independent variables and, hence, include only independent variables (omitting length of stay and guideline compliance). In Table 3, we estimate structural models that estimate separate direct and indirect pathways by which bedservice units may influence costs.

The first two columns of Table 2 present the full and parsimonious index stay cost models, respectively. The parsimonious models retain only those regressors with $p$-values of 0.20 or lower in the full models in an effort to estimate coefficients with greater precision. Table 2 includes only the index stay VHA cost models, because we
Table 1.

Descriptive statistics for poststroke patients receiving care in acute rehabilitation bedservice units (ARBUs) versus subacute rehabilitation bedservice units (SRBUs) in Department of Veterans Affairs medical centers.

\begin{tabular}{lcc}
\hline \multicolumn{1}{c}{ Variable } & \multicolumn{2}{c}{ Frequency (\%) or Mean \pm SD } \\
\cline { 2 - 3 } & $\begin{array}{c}\text { ARBUs } \\
(\boldsymbol{n}=\mathbf{3 1 5})\end{array}$ & $\begin{array}{c}\text { SRBUs } \\
(\boldsymbol{n}=\mathbf{1 6 8})\end{array}$ \\
\hline Race: White & $187(60.1)$ & $102(60.7)$ \\
Sex: Male & $300(97.6)$ & $164(96.5)$ \\
Marital Status: Married & $159(53.0)$ & $79(51.1)$ \\
Impairment Code & & \\
Left-Body Involvement & $133(42.8)$ & $69(41.1)$ \\
Right-Body Involvement & $135(43.4)$ & $70(41.7)$ \\
Bilateral Movement & $16(5.1)$ & $4(2.4)$ \\
No Paresis & $8(2.6)$ & $5(3.0)$ \\
Other Stroke & $19(6.1)$ & $20(11.9)$ \\
Age (yr) & $68.45 \pm 11.30$ & $68.85 \pm 10.90$ \\
Onset to Admit (d) & $26.29 \pm 103.80$ & $45.71 \pm 308.80$ \\
Compliance & $75.3 \pm 9.8$ & $74.0 \pm 11.9$ \\
Length of Stay (d) & $21.7 \pm 15.6$ & $28.6 \pm 19.6$ \\
FIM Admission Score & & \\
Cognitive & & \\
Motor & $24.21 \pm 7.60$ & $21.30 \pm 8.40$ \\
Total & $46.88 \pm 16.30$ & $44.07 \pm 19.00$ \\
Cost (\$) & $71.08 \pm 21.30$ & $65.38 \pm 24.50$ \\
Total Index & & \\
Total Short-Term & $16,320 \pm 18,431$ & $17,865 \pm 21,151$ \\
Total Long-Term & $38,252 \pm 56,329$ & $34,363 \pm 53,982$ \\
Total 2-year & $74,597 \pm 63,312$ & $72,152 \pm 62,947$ \\
Components of Cost (\$) & & \\
Nursing & $10,196 \pm 16,409$ & $10,046 \pm 10,914$ \\
Pharmacy & $1,803 \pm 3,193$ & $2,269 \pm 5,522$ \\
Surgical & $1,067 \pm 3,867$ & $1,709 \pm 6,032$ \\
Laboratory & $699 \pm 1,141$ & $724 \pm 1,041$ \\
All Other & & \\
\hline Note: $\chi^{2}$ test for categorical variables; -test for continuous variables. \\
F $\leq$ 0.05. & & \\
FIM = Functional Independence Measure, SD = standard deviation. \\
\hline \hline
\end{tabular}

were unable to detect any statistically significant differences between ARBUs and SRBUs for short-term, longterm, and total 2-year costs. We used OLS estimation in place of generalized linear model estimation, as suggested by the testing procedures recommended by Manning and Mullahy [16]. Also, we found that a square root transformation performed better in terms of reducing skewness and kurtosis than the natural log transformation used in most cost models. For this reason, we present in Table 2 both the untransformed and square root-transformed cost models for both full and parsimonious specifications. 
Table 2.

Regression models for index rehabilitation stay costs.

\begin{tabular}{|c|c|c|c|c|}
\hline Variable & $\begin{array}{c}\text { Full Cost } \\
\left(R^{2}=0.40\right)\end{array}$ & $\begin{array}{l}\text { Parsimonious Cost } \\
\qquad\left(R^{2}=0.39\right)\end{array}$ & $\begin{array}{l}\text { Full Square Root Cost } \\
\qquad\left(R^{2}=0.41\right)\end{array}$ & $\begin{array}{l}\text { Parsimonious Square } \\
\text { Root Cost }\left(R^{2}=0.40\right)\end{array}$ \\
\hline ARBU & $-5,979.90^{*}$ & $-5,850.79^{*}$ & $-14.87^{\dagger}$ & $-14.58^{\dagger}$ \\
\hline \multicolumn{5}{|l|}{ Impairment Code } \\
\hline Left-Body Involvement & 765.07 & - & 2.22 & - \\
\hline Right-Body Involvement & $1,866.22$ & - & 7.11 & - \\
\hline Bilateral Movement & $-2,693.24$ & - & -13.70 & - \\
\hline No Paresis & -183.92 & - & -1.90 & - \\
\hline Other Stroke & Reference & - & Reference & - \\
\hline Onset to Admit & -0.27 & - & 0.004 & - \\
\hline \multicolumn{5}{|l|}{ FIM at Admission } \\
\hline Motor & $-451.23^{*}$ & $-475.41^{*}$ & $-1.38^{*}$ & $-1.49^{*}$ \\
\hline Cognitive & -75.75 & - & -0.34 & \\
\hline Age & $-204.07^{*}$ & $-191.82^{*}$ & $-0.55^{*}$ & $-0.49^{*}$ \\
\hline Married & 41.99 & - & 1.14 & - \\
\hline White & $1,390.77$ & - & 1.83 & - \\
\hline \multicolumn{5}{|l|}{ VAMC Site } \\
\hline 516 & $-7,382.23^{\dagger}$ & $-6,105.27$ & -20.97 & -17.58 \\
\hline 523 & 2,029.84 & $1,252.22$ & 9.38 & 4.99 \\
\hline 528 & $8,397.23^{\dagger}$ & $9,106.78^{*}$ & $25.13^{\dagger}$ & $27.14^{*}$ \\
\hline 546 & $-5,887.32$ & $-5,975.21$ & -17.64 & -18.37 \\
\hline 549 & $8,993.35^{*}$ & $9,848.49^{*}$ & 21.45 & $24.43^{\dagger}$ \\
\hline 552 & $-4,486.92$ & $-3,496.23$ & -18.55 & -15.20 \\
\hline 553 & $-6,194.42$ & $-6,406.22$ & -17.63 & -17.28 \\
\hline 578 & 4,128.98 & 4,223.46 & 4.59 & 4.30 \\
\hline 583 & $12,709.93^{*}$ & $13,071.03^{*}$ & $36.97^{*}$ & $37.44^{*}$ \\
\hline 598 & $7,304.03^{*}$ & $7,487.36^{*}$ & 17.23 & 17.55 \\
\hline 600 & -890.91 & -550.78 & -4.63 & -3.14 \\
\hline 605 & $-6,843.06$ & $-6,053.16$ & -20.45 & -17.45 \\
\hline 618 & $2,855.75$ & 3,695.13 & 8.75 & 10.58 \\
\hline 630 & 6,627.13 & 6,926.72 & 20.72 & 21.85 \\
\hline 635 & $-7,677.20^{\dagger}$ & $-7,466.79^{\dagger}$ & $-30.90^{*}$ & $-30.83^{*}$ \\
\hline 642 & $-4,266.90$ & $-4,502.05$ & -25.27 & -24.45 \\
\hline 648 & $-2,015.00$ & -896.68 & -4.96 & -1.99 \\
\hline 671 & $-1,291.84$ & -546.89 & -17.32 & -11.93 \\
\hline 672 & -790.60 & $-1,013.02$ & -9.21 & -7.89 \\
\hline 673 & $-2,017.43$ & $-1,110.00$ & -5.40 & -2.52 \\
\hline 678 & $-4,072.49$ & $-3,580.50$ & -9.24 & -7.84 \\
\hline 688 & 2,628.07 & 2,907.72 & -3.40 & -0.94 \\
\hline 691 & Reference & - & Reference & - \\
\hline
\end{tabular}

Table 2 suggests that ARBUs had significantly lower index rehabilitation stay VHA costs than SRBUs by a magnitude of approximately $\$ 6,000$, or roughly 25 percent of mean index stay VHA costs in SRBUs. This result appears in all four regressions presented in Table 2 and is statistically significant at the 0.05 level in the untransformed models and at the 0.10 level in the square root-transformed model. In addition, higher motor functioning at admission, as measured by higher admission motor FIM score, was associated with lower index stay 
JRRD, Volume 47, Number 5, 2010

Table 3.

Structural models of regressions for cost, length of stay, and guideline compliance score.

\begin{tabular}{|c|c|c|c|c|}
\hline Variable & $\begin{array}{l}\text { Untransformed Cost } \\
\left(R^{2}=0.74\right)\end{array}$ & $\begin{array}{l}\text { Square Root Cost } \\
\qquad\left(R^{2}=0.69\right)\end{array}$ & $\begin{array}{c}\text { Natural Log Length } \\
\text { of Stay }\left(R^{2}=0.48\right)\end{array}$ & $\begin{array}{l}\text { Guideline Compliance } \\
\text { Score }\left(R^{2}=\text { undefined }\right)\end{array}$ \\
\hline ARBU & -249.13 & -0.18 & $-0.34^{*}$ & $5.49^{*}$ \\
\hline Length of Stay & $740.18^{*}$ & $2.06^{*}$ & - & $0.18^{*}$ \\
\hline Guideline Compliance & $103.26^{*}$ & $0.45^{*}$ & - & - \\
\hline \multicolumn{5}{|l|}{ Score } \\
\hline \multicolumn{5}{|l|}{ Impairment Code } \\
\hline Left-Body Involvement & -608.47 & -0.34 & 0.16 & 0.34 \\
\hline Right-Body Involvement & 354.55 & 3.98 & 0.15 & 1.87 \\
\hline Bilateral Movement & $-2,132.88$ & -10.71 & -0.05 & -2.40 \\
\hline No Paresis & $-1,437.60$ & 0 & $0.28^{\dagger}$ & -2.43 \\
\hline Other Stroke & Reference & Reference & Reference & Reference \\
\hline Onset to Admit & 2.05 & 0.01 & 0 & $0.004^{\dagger}$ \\
\hline \multicolumn{5}{|l|}{ FIM at Admission } \\
\hline Motor & $-140.42^{*}$ & $-0.51^{*}$ & $-0.02^{*}$ & 0.02 \\
\hline Cognitive & -37.29 & -0.25 & 0 & 0.03 \\
\hline Age & $-93.11^{*}$ & $-0.22^{\dagger}$ & $-0.004^{\dagger}$ & $-0.08^{*}$ \\
\hline Married & 804.01 & 2.91 & -0.04 & 0.13 \\
\hline White & 624.75 & -0.74 & -0.01 & 0.60 \\
\hline \multicolumn{5}{|l|}{ VAMC Site } \\
\hline 516 & $-8,126.58^{*}$ & $-24.18^{*}$ & 0.18 & $8.32^{*}$ \\
\hline 523 & -126.73 & 5.66 & 0.23 & -5.12 \\
\hline 528 & 2,305.49 & 10.33 & $0.55^{*}$ & $-10.59^{*}$ \\
\hline 546 & $-8,267.42^{*}$ & $-22.43^{*}$ & $0.35^{*}$ & -2.36 \\
\hline 549 & $-15,673.59^{*}$ & $-44.72^{*}$ & $1.00^{*}$ & $-8.17^{*}$ \\
\hline 552 & $-15,083.31^{*}$ & $-45.77^{*}$ & $0.55^{*}$ & $-13.61^{*}$ \\
\hline 553 & $-8,094.40^{*}$ & $-22.27^{\dagger}$ & 0.31 & -4.87 \\
\hline 578 & -890.66 & -10.40 & $0.27^{\dagger}$ & $6.61^{*}$ \\
\hline 583 & $7,357.31^{*}$ & $23.09^{*}$ & $0.31^{\dagger}$ & -4.10 \\
\hline 598 & $-1,573.12$ & -6.49 & $0.57^{*}$ & -3.21 \\
\hline 600 & -739.87 & -2.86 & 0.07 & -4.34 \\
\hline 605 & $-1,629.05$ & -4.36 & -0.09 & $-10.56^{*}$ \\
\hline 618 & $-2,972.35$ & -8.60 & $0.38^{*}$ & $9.98^{*}$ \\
\hline 630 & 1,992.28 & 7.78 & 0.24 & 2.32 \\
\hline 635 & $-5,530.72^{*}$ & $-22.89^{*}$ & -0.08 & $-8.97^{*}$ \\
\hline 642 & $-6,695.73^{\dagger}$ & $-26.91^{*}$ & $0.38^{*}$ & $-14.48^{*}$ \\
\hline 648 & $-7,887.91^{*}$ & $-21.34^{*}$ & $0.54^{*}$ & 1.94 \\
\hline 671 & $-20,327.52^{*}$ & $-68.75^{*}$ & $0.51^{*}$ & $-10.12^{*}$ \\
\hline 672 & $-6,833.02^{*}$ & $-25.66^{*}$ & $0.42^{*}$ & -0.52 \\
\hline 673 & $-4,857.96^{\dagger}$ & -12.17 & $0.41^{*}$ & -3.57 \\
\hline 678 & $-12,863.84^{*}$ & $-34.62^{*}$ & $0.64^{*}$ & $7.36^{*}$ \\
\hline 688 & -759.13 & -12.95 & 0.12 & $5.99^{\dagger}$ \\
\hline 691 & Reference & Reference & Reference & Reference \\
\hline
\end{tabular}

costs. A 10-point increase in admission motor FIM score would correspond to $\$ 4,500$ to $\$ 4,750$ lower index stay costs, all other factors constant. Older age was also statis- tically significant across all four models and tended to reduce index stay costs. This somewhat surprising result may stem from older patients receiving less intensive 
rehabilitation services, after accounting for the influence of admission functional status. Finally, a number of the facility variables were statistically significant, reflecting the considerable facility variations in costs commonly found in other studies of inpatient costs [18]. The regressors listed in Table 2 accounted for approximately 40 percent of the variation in the cost-dependent variable.

The results presented in Table 2 indicate that the type of rehabilitation unit has a sizable influence on index rehabilitation stay costs. However, the results in Table 2 provide little insight into the pathways by which such an effect is produced. The results in Table 3 yield information about the magnitudes of these pathways (both direct and indirect effects) and give a more precise understanding of the total effects observed in Table 2.

The results in Table 3 show the direct effects of all the variables on costs, length of stay, and guideline compliance scores. We also used the key coefficients from Table 3 to calculate the magnitudes of the various indirect pathways whereby type of rehabilitation unit influences index stay VHA costs (see the Appendix for details on these calculations, available online only). ARBUs had their largest indirect effect on index rehabilitation stay VHA costs through reducing lengths of stay by approximately 8.2 days. Given the marginal cost per day of $\$ 740$, this translates into roughly $\$ 6,000$ in lower costs, which is essentially the entire total effect of type of rehabilitation unit on index stay VHA costs, whether measured in the reduced-form model from Table 2 or as the sum of the direct and indirect effects in Table 3. Once the impacts of unit type on lengths of stay and guideline compliance are included, we fail to find a statistically significant direct effect of unit type on index stay costs.

In addition to examining total index rehabilitation stay costs, we estimated separate regression models for the components of total costs as provided in the VHA DSS NDE data (nursing, pharmacy, laboratory, surgical, and all other), controlling for type of bed unit (ARBU vs SRBU), impairment code, onset to admission, admission motor and cognitive FIM scores, and demographics (age, marital status, and race). Fixed effects were also added to control for facility (the site variables listed in Table 3). These models demonstrate that ARBUs save approximately $\$ 3,500$ in nursing costs compared with SRBUs. ARBUs also demonstrate approximately $\$ 900$ lower pharmacy costs. We did not find statistically significant effects of type of rehabilitation unit on laboratory, surgical, or all other costs of the index rehabilitation stay.
In addition to reduced lengths of stay, ARBUs are also associated with higher rates of stroke rehabilitation guideline compliance $(+5.5$ points on the $0-100$ point scale). Guideline compliance, in turn, is associated with higher costs of care (by \$103 per guideline-compliance point). Longer lengths of stay are also associated with slightly higher guideline compliance scores $(+0.18$ more guideline-compliance points per extra day).

\section{DISCUSSION}

These results stand in contrast to the conventional wisdom that shifting care away from acute settings to subacute settings saves money. The evidence just presented suggests that the 8.3 days shorter mean length of stay in ARBUs compared with SRBUs is the primary source of the substantial $\$ 6,000$ lower VHA costs per stay in ARBUs observed in this study. The fact that nursing costs account for $\$ 3,500$ of these lower costs is consistent with reduced lengths of stay as the primary driver of these results. Moreover, the lower costs observed in ARBUs in this study combined with the higher rates of guideline compliance and improved outcomes in ARBUs found in previous work $[10,19]^{*}$ suggest that stroke rehabilitation in an ARBU may be more cost-effective than stroke rehabilitation in an SRBU.

We emphasize, however, that our cost results apply to the index stay only and that we were unable to find statistically significant cost impacts beyond the index stay. Also, our analyses apply only to costs and we did not examine clinical outcomes for the patients in our sample. Therefore, our analyses fall short of a full cost-effectiveness analysis of acute versus subacute stroke rehabilitation.

Our finding that higher rates of guideline compliance are associated with higher costs is noteworthy, but perhaps equally noteworthy is the small size of this effect. As shown in Table 3, a 1-point increase in the guidelinecompliance score is associated with an approximately $\$ 100$ increase in costs. Correspondingly, a substantial 10-point increase in guideline compliance would be associated with a relatively small $\$ 1,000$ increase in costs, only 4 percent

\footnotetext{
* Reker DM, Duncan PW. The process and outcomes of stroke care in the VHA. Department of Veterans Affairs, Veterans Health Administration, Rehabilitation Research and Development Service, grant O3131R; 2006.
} 
of the $\$ 25,000$ mean rehabilitation stay cost. To the extent that improved guideline compliance improves functional outcomes, this suggests that higher rates of guideline compliance may be desirable not only from a clinical perspective but also from a financial perspective.

This suggestion is tempered, however, by several limitations in the present study: (1) we were unable to detect statistically significant differences is short-term, long-term, or 2-year annual VHA costs, leaving open the question of whether possible postdischarge increases in costs for patients treated in ARBUs offset the lower costs observed during the index rehabilitation stay (the results remained inconclusive when we examined these longer-term costs excluding the costs of the index stay); (2) our data pertain only to VHA stroke patients and are therefore limited in their scope, since both ARBUs and SRBUs treat a wide variety of rehabilitation patients, including those with spinal cord injuries, amputations, and traumatic brain injuries; (3) this study, by necessity, only examined the costs associated with VHA use and omitted any use of non-VHA care, thereby making this analysis more of a business case analysis from the VHA perspective rather than societal perspective (prior research among stroke patients has shown substantial amounts of poststroke Medicare utilization among Medicare-enrolled veterans [19]); and (4) our use of observational data raises the possibility that our results may exhibit statistical selection bias whereby those patients who are selected for treatment in a particular type of unit (ARBU or SRBU) differ from other patients in unobserved ways that influence the outcomes under study. (For example, if clinicians tend to direct more motivated stroke patients to ARBUs and less motivated stroke patients to SRBUs, our failure to measure motivation directly could bias our results in favor of ARBUs.) While selection bias is always a danger in observational studies, we do not believe that selection bias alone could account for the results observed here for several reasons. First, we were able to control for patient functional status on admission (using admission motor and cognitive FIM scores), thereby eliminating or at least reducing a major potential source of selection bias. Next, our data enabled us to include most of the other important covariates (age, sex, race, marital status, time since onset, impairment code, etc.) found in observational studies of this type and descriptive comparisons of patient characteristics revealed broad substantial similarities in patient characteristics across ARBUs and SRBUs. Also, the sizable effects observed in our models for index stay VHA costs and lengths of stay suggest fundamental differences in duration and intensity of rehabilitation between ARBUs and SRBUs that extend well beyond patient selection. Finally, the VHA facilities in this study typically had only one type of VHA rehabilitation unit (acute or subacute), meaning that any bias in the selection of patients between the two type of units would require a patient transfer to another (often remote) facility.

\section{CONCLUSIONS}

Our results suggest that the VHA should proceed cautiously in substituting SRBU care for ARBU care. Although the conventional wisdom is that hospital settings are more expensive than nonhospital settings, our results show that ARBU costs per index stroke rehabilitation stay are \$6,000 lower than comparable SRBU costs. Additional research is required before definitive conclusions can be drawn regarding the cost-effectiveness of poststroke rehabilitation in ARBUs versus SRBUs. We are presently conducting a cost-utility study funded through the VA Rehabilitation Research and Development Service that examines a broader array of stroke rehabilitation structures and also includes measures of rehabilitation outcomes. This work should improve our understanding of the relationships between structure, process, outcomes, and costs in stroke rehabilitation and provide better guidance to policymakers for improving the effectiveness and efficiency of VHA stroke rehabilitation services.

\section{ACKNOWLEDGMENTS}

\section{Author Contributions:}

Study concept and design: W. B. Vogel, D. Reker.

Acquisition of data: D. Reker.

Analysis and interpretation of data: W. B. Vogel, T. E. Barnett, D. Reker. Drafting of manuscript: T. E. Barnett, W. B. Vogel.

Critical revision of manuscript for important intellectual content:

D. Reker, T. E. Barnett, W. B. Vogel.

Statistical analysis: W. B. Vogel, T. E. Barnett.

Obtained funding: W. B. Vogel, D. Reker.

Financial Disclosures: The authors have declared that no competing interests exist.

Funding/Support: This material was based on work supported by the VHA Health Services Research and Development Service (grant RRP 06-184, "VHA costs of acute vs subacute rehabilitation care for stroke.”) Additional Contributions: Dr. Reker is presently a consultant to the VHA Rehabilitation Outcomes Research Enhancement Award Program, Gainesville, Florida. 


\section{REFERENCES}

1. Martínez-Vila E, Irimia P. The cost of stroke. Cerebrovasc Dis. 2004;17 Suppl 1:124-29. [PMID: 14694289] DOI:10.1159/000074804

2. Agency for Health Care Policy and Research. Clinical practice guidelines: Post-Stroke Rehabilitation. Rockville (MD): Department of Health and Human Services; 1995.

3. Thom T, Haase N, Rosamond W, Howard VJ, Rumsfeld J, Manolio T, Zheng ZJ, Flegal K, O’Donnell C, Kittner S, Lloyd-Jones D, Goff DC Jr, Hong Yuling. Heart Disease and Stroke Statistics - 2006 Update. Dallas (TX): American Heart Association; 2006.

4. VHA office of Finance Allocation Resource Center. Annual Workload and Cost Report-FY05 [Internet]; 2006 [cited 2010 Apr 9]. Available from:

http://vaww.arc.med.va.gov/vapublic/ arci_sr4v3.asp?sDTP=06\&sSO=2006FY./

5. Palmer AJ, Valentine WJ, Roze S, Lammert M, Spiesser J, Gabriel S. Overview of costs of stroke from published, incidence-based studies spanning 16 industrialized countries. Curr Med Res Opin. 2005;21(1):19-26. [PMID: 15881472] DOI:10.1185/030079904X17992

6. Wagner TH, Richardson SS, Vogel B, Wing K, Smith MW. Cost of inpatient rehabilitation care in the Department of Veterans Affairs. J Rehabil Res Dev. 2006;43(7):929-38. [PMID: 17436178] DOI:10.1682/JRRD.2005.10.0162

7. Hayes J, Vogel WB, Reker DM. Factors associated with VHA costs of care for first 12 months after first stroke. J Rehabil Res Dev. 2008;45(9):1375-84. [PMID: 19319761] DOI:10.1682/JRRD.2007.09.0157

8. Kizer KW. From the Veterans Health Administration. JAMA. 1995;273(23):1817. [PMID: 7776487]

DOI:10.1001/jama.273.23.1817

9. Zinn S, Dudley TK, Bosworth HB, Hoenig HM, Duncan PW, Horner RD. The effect of poststroke cognitive impairment on rehabilitation process and functional outcome. Arch Phys Med Rehabil. 2004;85(7):1084-90. [PMID: 15241754] DOI:10.1016/j.apmr.2003.10.022

10. Duncan PW, Horner RD, Reker DM, Samsa GP, Hoenig H, Hamilton B, LaClair BJ, Dudley TK. Adherence to postacute rehabilitation guidelines is associated with functional recovery in stroke. Stroke. 2002;33(1):167-77. [PMID: 11779907] DOI:10.1161/hs0102.101014
11. LaClair BJ, Reker DM, Duncan PW, Horner RD, Hoenig H. Stroke care: A method for measuring compliance with AHCPR guidelines. Am J Phys Med Rehabil. 2001;80(3): 235-42. [PMID: 11237279] DOI:10.1097/00002060-200103000-00017

12. Keith RA, Granger CV, Hamilton BB, Sherwin FS. The Functional Independence Measure: A new tool for rehabilitation. Adv Clin Rehabil. 1987;1:6-18. [PMID: 3503663]

13. Uniform Data System for Medical Rehabilitation [Internet]. Buffalo (NY): Uniform Data Systems, 1999 [cited 2010 Feb 4]. Available from: http://www.udsmr.org/.

14. Phibbs C, Yu W, Barnett PG. Research guide to Decision Support System National Cost Extracts 1998-2004. Washington (DC): Department of Veterans Affairs; 2006.

15. Health Economics Resource Center. Estimating VA treatment cost: Methods and applications. Med Care Res Rev. 2003;60(3S).

16. Manning WG, Mullahy J. Estimating log models: To transform or not to transform? J Health Econ. 2001;20(4):461-94. [PMID: 11469231] DOI:10.1016/S0167-6296(01)00086-8

17. Park R. Estimation with heteroscedastic error terms. Econometrica. 1966;34(4):888. DOI:10.2307/1910108

18. Vogel WB, Rittman M, Bradshaw P, Nissen D, Anderson L, Bates B, Marshall C. Outcomes from stroke rehabilitation in Veterans Affairs rehabilitation units: Detecting and correcting for selection bias. J Rehabil Res Dev. 2002;39(3): 367-83. [PMID: 12173757]

19. Jia H, Zheng Y, Reker DM, Cowper DC, Wu SS, Vogel WB, Young GC, Duncan PW. Multiple system utilization and mortality for veterans with stroke. Stroke. 2007;38(2): 355-60. [PMID: 17194888$]$ DOI:10.1161/01.STR.0000254457.38901.fb

Submitted for publication July 9, 2009. Accepted in revised form February 4, 2010.

This article and any supplementary material should be cited as follows:

Vogel WB, Barnett TE, Reker D. Cost differences in VHA acute and subacute rehabilitation units: Implications for VHA resource planning. J Rehabil Res Dev. 2010;47(5):431-40.

DOI:10.1682/JRRD.2009.07.0094. 
